\title{
Bridging the Radiotherapy Education Gap in Africa: Lessons Learnt from the Cape Town Access to Care Training Programme Over the Past 5 Years (2015-2019)
}

\author{
Hester Burger ${ }^{1}$ (D) $\cdot$ Bridget Wyrley-Birch ${ }^{2} \cdot$ Nanette Joubert $^{1} \cdot$ Christoph Jan Trauernicht $^{1,3} \cdot$ Jose-Manuel Valentim $^{4}$. \\ Jens Groll ${ }^{4}$ - Stefan Berz ${ }^{4} \cdot$ Natalia Vowles $^{2}$. Jeannette Parkes ${ }^{5}$
}

Accepted: 12 April 2021 / Published online: 29 April 2021

(c) American Association for Cancer Education 2021

\begin{abstract}
The role of radiotherapy (RT) in cancer care is well described, with a clear correlation between access to radiotherapy and overall survival. Cancer mortality rates in Africa are substantially higher than those of the rest of the world, which may be partly attributed to lack of RT access and insufficient human resources. The Access to Care (A2C) Cape Town RT training programme was created in 2014 with the aim of supplementing practical RT training in the region, focusing on clinics moving from 2 to 3D conformal radiotherapy (3DCRT). The programme makes use of hybrid teaching methods, including pre-course e-learning followed by 17 on-site days of free-thinking design exercises, didactic learning, hands-on treatment planning computer sessions (39\% of total teaching time), virtual simulation training and departmental demonstration sessions. Email support is offered to all teams for 3 months after each course to develop clinical protocols. Thirteen teams (radiation oncologist, medical physicist and radiation therapy technologist) from Africa attended the course between 2015 and 2019, with additional participants from seven South African and four international centres. E-learning done on the LäraNära training platform was only successful once formal progress tracking was introduced in 2019 (34\% vs. 76\% test completion rate). Delays between course attendance and initial clinical use of equipment proved to be detrimental to knowledge retention, with some centres having to send a second team for training. The course will be modified for remote teaching in 2021 , to make provision for the global changes in travel due to Covid-19.
\end{abstract}

Keywords Radiotherapy training $\cdot$ Hybrid learning $\cdot$ E-learning $\cdot$ Virtual reality platform $\cdot$ Treatment planning $\cdot$ LMIC $\cdot 3 \mathrm{D}$ conformal radiotherapy $\cdot$ Africa

\section{Introduction}

Making appropriate cancer care available to all patients has become a worldwide priority. This is because the incidence

Hester Burger

Hester.Burger@uct.ac.za

1 Division of Medical Physics, Groote Schuur Hospital and University of Cape Town, Anzio Road, Observatory, Cape Town, South Africa

2 Department of Radiation Technology, Cape Peninsula University of Technology, Cape Town, South Africa

3 Present Address: Division of Medical Physics, Tygerberg Hospital and University of Stellenbosch, Cape Town, South Africa

4 Varian Medical Systems, Steinhausen, Switzerland

5 Department Radiation Oncology, Groote Schuur Hospital and University of Cape Town, Cape Town, South Africa of cancer worldwide is rapidly rising. Statistics from the Global Cancer Incidence, Mortality and Prevalence database (GLOBOCAN) show a 20\% increase from the 2015 estimates of 15 million new cases per year to 18 million cases in 2018, and a further $9 \%$ increase to 19.3 million new cases estimated for 2020 [1-4]. The GLOBOCAN 2020 report further highlights the inequity in health care between higher and lower resource settings, predicting that countries classified as low or medium Human Development Index (HDI) regions will have the highest relative increase in cancer incidence between 2020 and 2040. The number of new cases in low HDI countries is estimated to rise by $96 \%$ in this time period, compared to a $64.7 \%$ increase in medium HDI, a 
$56.2 \%$ increase in high HDI and only a $32.3 \%$ increase in very high HDI countries [3].

Although the upward trend in both incidence and cancers deaths is clear from the reports, care must be taken with the interpretation of the data due to limited access to populationbased cancer registries on the African continent. Of the 46 countries in sub-Saharan Africa, only 25 are reported to have population-based cancer registries. Even in the countries where the registries exist, data is often incomplete, and the actual incidence and mortality are likely to be higher than reported. [5]

The role of radiotherapy in the management of cancer was well described by the Lancet Oncology Commission Global Task Force on Radiotherapy for Cancer Control (GTFRCC) report in 2015, showing that approximately half of all oncology patients will require radiotherapy at some point during their treatment [6]. The contribution of radiotherapy to survival is estimated at around $40 \%$, and a clear correlation can be seen between the number of machines available per million population and the cancer mortality incidence rate (CMI) [7].

Currently, cancer death rates in Africa are reported to be higher than those observed in many other regions. GLOBOCAN 2020 statistics show a cancer incidence of 1,109 million new cases per year for the African continent, with 711 429 deaths (64.1\% deaths relative to incidence), compared to the world statistics of $19,293 \mathrm{~m}$ cases and $9.958 \mathrm{~m}$ deaths (51.6\% deaths relative to incidence) [2].

The reasons for this are complex; however, since radiotherapy is a key treatment modality for cancer, the higher cancer death rate in Africa may be partly attributable to lack of access to quality radiotherapy services, requiring both sufficient number of machines and qualified staff to operate the units $[7,8]$.

With half of all cancer patients requiring radiotherapy, the estimated African patient load is more than 550, 000 radiotherapy patients per year $[1-3,6]$. However, the International Atomic Energy Agency's (IAEA) Directory of Radiotherapy Centres (DIRAC) database shows that in 2021 there were only 412 external beam radiotherapy treatment units available in Africa, with an additional 102 brachytherapy units reported [9]. The IAEA recommends that one tele-radiotherapy machine be available for every 250,000 population; however, Africa only has one machine available for every 3.56 million people [7, 9]. In addition, the data from 2021 show that 223 (54\%) of these units were in either Egypt or South Africa, thus highlighting the dire situation in many other African countries [9]. Machine availability in Africa, measured as megavoltage treatment unit per million population, decreased in the time period 2010 to 2013 ( 0.27 vs. $0.25 \mathrm{MV}$ RT units/million population), as ageing machines started to fail, and replacement and maintenance programmes could not keep up with the increase in population. The situation did improve slightly from 2017 to 2021 (312 vs. 412 MV RT units; 0.25 vs. 0.30 MV RT units/ million population), with Cobalt-60 teletherapy machines being replaced with more modern linear accelerators $(25 \%$ of machines were Co60 in 2017 vs. 16\% in 2020) and more than half of units reported to be 10 years or younger [9-12].

Human resource availability further aggravates the situation. In 2014, it was estimated that in low- and middleincome countries (LMIC), an additional 12, 960 radiation oncologists, 6480 medical physicists, 3240 dosimetrists and 20, 520 radiation therapists (RTTs) would be required to staff the available machines. [12] With the increase in both machine numbers and the increased complexity of machines and treatment techniques since 2013, several global initiatives were launched to address the critical staff shortage as well as the skills shortage in Africa. These included collaborations between governmental and non-governmental organizations, industry partners and academic institutions. Various teaching platforms were created and used to promote modern hybrid and e-learning platforms to expand the reach of such programmes $[13,14]$. One of the initiatives was the Access to Care (A2C) Cape Town radiotherapy training programme, launched in 2014 with the specific aim of supplementing practical radiotherapy training on the African continent and thus increasing skills of clinical radiotherapy staff $[15,16]$.

The project was launched as a collaboration between the University of Cape Town (UCT), Cape Peninsula University of Technology (CPUT) and Varian Medical Systems (VMS) and aimed to assist qualified radiotherapy professionals in English-speaking regions in Africa to safely move from basic radiotherapy techniques (2D radiotherapy) to modern 3D conformal radiotherapy techniques (3DCRT) on linear accelerators. It was not designed to be accredited as part of a formal training programme but rather aimed to supplement existing programmes.

The A2C programme was set up under the umbrella of the global Varian Access to Care education initiative which was created to bridge the radiotherapy education gaps in regions where these specific training needs were identified. Although radiotherapy education programmes are well established in the larger academic centres in South Africa, the same cannot be said of many smaller South African centres as well as other sub-Saharan African countries; thus, the A2C programme was created to address the need in these areas [12].

As a multi-university/industry collaboration, a main contract and five separate memorandums of understanding were set up to ensure compliance with good financial and clinical governance principles, both in terms of South African as well as international law. To prevent additional human resource pressure on the local faculty, the programme also included budget to fund one part-time medical physicist (UCT) as well as one part-time radiation therapy technologist (CPUT). [15, 16] 
The aim of this paper is to report on the activities of the Access to Care Cape Town radiotherapy training programme for the time period 2015-2019.

\section{Materials and Methods}

This study was undertaken to review the first 5-year term of the A2C Cape Town programme in terms of:

- Educational instruments and platform

- Teaching methodology and programme design

- Geographic reach of the programme

- Programme evaluation and participant feedback

In addition, the geographic reach of the programme was assessed and compared to local machine availability and age as reported by the Directory of Radiotherapy Centres international database [9].

\section{Ethical Issues}

Ethics approval for the study was obtained from the Human Research Ethics Committee of the University of Cape Town (HREC REF: 829/2019). Informed consent was obtained from participants to use feedback comments anonymously for reports and study purposes.

\section{Results}

\section{Educational Instruments and Platform}

The A2C training programme was set up as a hybrid e-learning and in-classroom teaching model with access to modern digital classrooms, a virtual reality radiotherapy simulation system, radiotherapy treatment planning computer access and demonstrations in the clinical environment.

Two existing classrooms were repurposed and modernized to accommodate a VERT ${ }^{\mathrm{TM}}$ radiotherapy simulation system as well as a cloud based Varian Eclipse Treatment Planning System (TPS) laboratory.

\section{VERT ${ }^{\mathrm{TM}}$ Radiotherapy Simulation System}

The VERT ${ }^{\mathrm{TM}}$ system was the first installation in Africa, allowing for 2- or 3-dimensional visualization of radiotherapy and medical physics quality assurance (QA) equipment. Participants with limited experience in operating linear accelerators can practice on the system, with no time pressure or risk of damage to the clinical equipment. In addition to simulation of normal operation of the equipment, the system allows for deliberate fault scenario programming.
Participants are encouraged to improve their critical thinking skills to find causes of quality assurance failures and patient setup errors. It is also possible to visualize and assess the internal dose changes and clinical consequences of these errors.

\section{Eclipse $^{\mathrm{TM}}$ Treatment Planning System (TPS) Laboratory}

Treatment planning computers are generally very high cost items, prohibiting teaching and training of students on a large scale. Access to clinical TPSs adds pressure on departments and increases the risk of students accidentally causing errors on the clinical system. To overcome these issues, a more cost-effective cloud-based solution was implemented.

Communication between local Dell Wyse Thin Client ${ }^{\mathrm{TM}}$ workstations and the main Varian server in Switzerland is achieved using the West Africa Cable system (WACS) through an agreement with the South African National Research and education Network (SANReN), as part of the University of Cape Town's quota. This allows access to Varian Eclipse TPSs for a maximum of 15 users at the facility.

\section{Additional Classroom Resources}

A key concern in many departments is lack of understanding of the radiotherapy departmental design and procurement process. Mobile magnetic white boards were added to all classrooms to allow students to take part in free-thinking departmental design exercises (Fig. 1). Every team is allocated a budget and infrastructure scenario at random and is then required to design a department based on this scenario. Teams can "purchase" items from the A2C shop, including RT machines, immobilization devices, computer and networking equipment, as well as quality assurance equipment. All boards are presented by the teams and then evaluated at the end of the course, encouraging teams to develop a

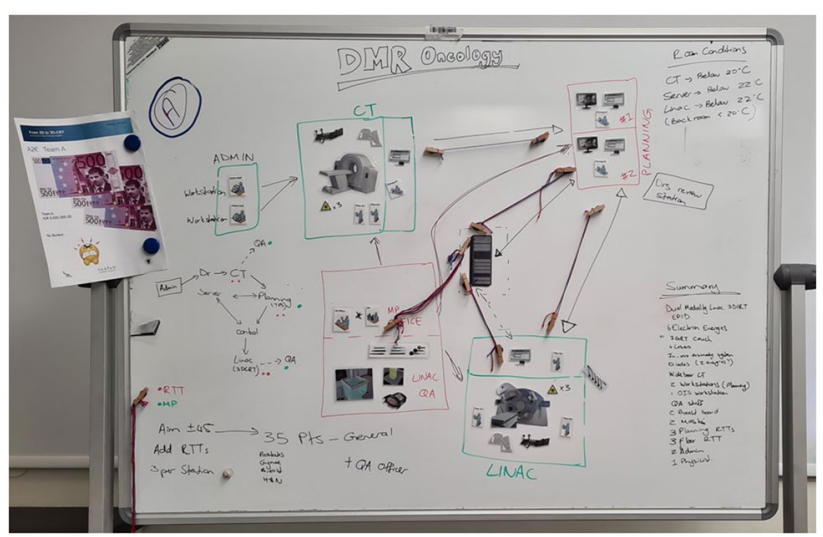

Fig. 1 Departmental design and procurement white board exercise 
better understanding of the implications of equipment and QA device choices on the functioning of the department.

In addition, eBeam ${ }^{\mathrm{TM}}$ detectors and software were installed as a cost-effective way to convert standard classroom walls into interactive whiteboards. Classroom capabilities were further enhanced in 2018 to allow for automated video recording in the VERT ${ }^{\mathrm{TM}}$ and Eclipse classrooms, automatically synchronizing video and slide feeds for future upload to the UCT VULA ${ }^{\mathrm{TM}}$ e-learning platform.

\section{Teaching Methodology and Programme Design}

\section{Overview of Programme}

The original focus of the training programme was to allow participants to develop the necessary skills to move from basic 2D radiotherapy to more advanced 3D conformal radiotherapy. Five clinical disease sites were included in the training programme, based on most prevalent cancers seen in the region. This included prostate, cervix, breast, head and neck and neurological lesions.

Participating centres were invited to send a team consisting of one radiation oncologist, one medical physicist and one RTT.

The curriculum that was implemented followed a modified version of the radiotherapy treatment chain for each clinical site [17]. Each element in the chain was defined in terms of the level of knowledge that each profession would need to be functional, distinguishing between.

- Broad-based knowledge elements requiring an understanding of the impact of choices made by the various professions in the department

- A deeper level of knowledge to allow for effective daily operation at a practical level

- Specialist level skills aimed at each specific profession.

As a result of the move towards integration of knowledge across the professions rather than communication of knowledge between the professions, the initial programme design of 2-week medical physics teaching followed by 2 weeks of clinical teaching was modified to allow for 3 weeks of teaching with all professions (radiation oncologists, medical physicists and radiation therapists) on-site for the full 17-day training period. This was followed by 3 months of email-based mentorship and support to allow the teams to develop clinical protocols for their own sites.

In 2018, a review of the programme and curriculum design was done based on feedback from participants. The feedback highlighted the need for more hands-on planning time to master technical planning skills. As a result, the programme was modified to only include prostate, cervix and breast cancer in the 17-day course period, with the remaining clinical sites to be addressed as separate courses. Hands-on time was increased, and individual and team assessment strategies were implemented to track progress during the course.

\section{Pre-course Online Training}

In March 2017, pre-course online training was added for all participants, using the LäraNära training platform, available through Varian education [14]. Access to care participants was granted access for this purpose to the Swedish-based radiotherapy distance education platform and learning management system [available at: https://www.laranara.se/en. html]. Participants were asked to complete eight modules on basic radiotherapy equipment, radiotherapy beam characteristics, principles of imaging, contouring, treatment planning, delivery techniques and radiation protection, including evaluation tests for each module. Each module requires approximately $15 \mathrm{~min}$ to complete, while the tests consisted of multiple-choice questions.

An assessment was done on the mean test completion rate for all participants. The completion rate for the first four courses was only $33.75 \%$ with many participants not completing any modules even though it was considered compulsory. In order to address this, a system of tracking and frequent personal reminders was implemented for the last two courses, resulting in a mean test completion rate for $76 \%$.

\section{Clinical Protocol Development}

The training course was originally designed to include development of clinical treatment protocols for prostate, cervix, breast, head and neck cancers as well as neurological cancers. Teams were guided during the course to develop and present at least one clinical protocol and then supported via email to develop the remaining protocols during the 3-month mentorship period.

Due to a wide variation in the style and quality of the protocols received, the decision was taken in 2017 to adopt the protocol template developed by the Swedish Radiation Safety Authority [18]. In addition, the number of protocols to be developed was reduced to only include prostate, cervix and breast as clinical sites, in line with the change in programme implemented in 2018.

\section{Teaching Methodology Utilization}

An analysis was done to investigate the time allocated to different teaching methodologies for each course run between 2015 and 2019. An assessment of the number of hours dedicated to each type of teaching methodology is given in Fig. 2. 
Fig. 2 Teaching methodology utilization (hours per course): 2015 to 2019

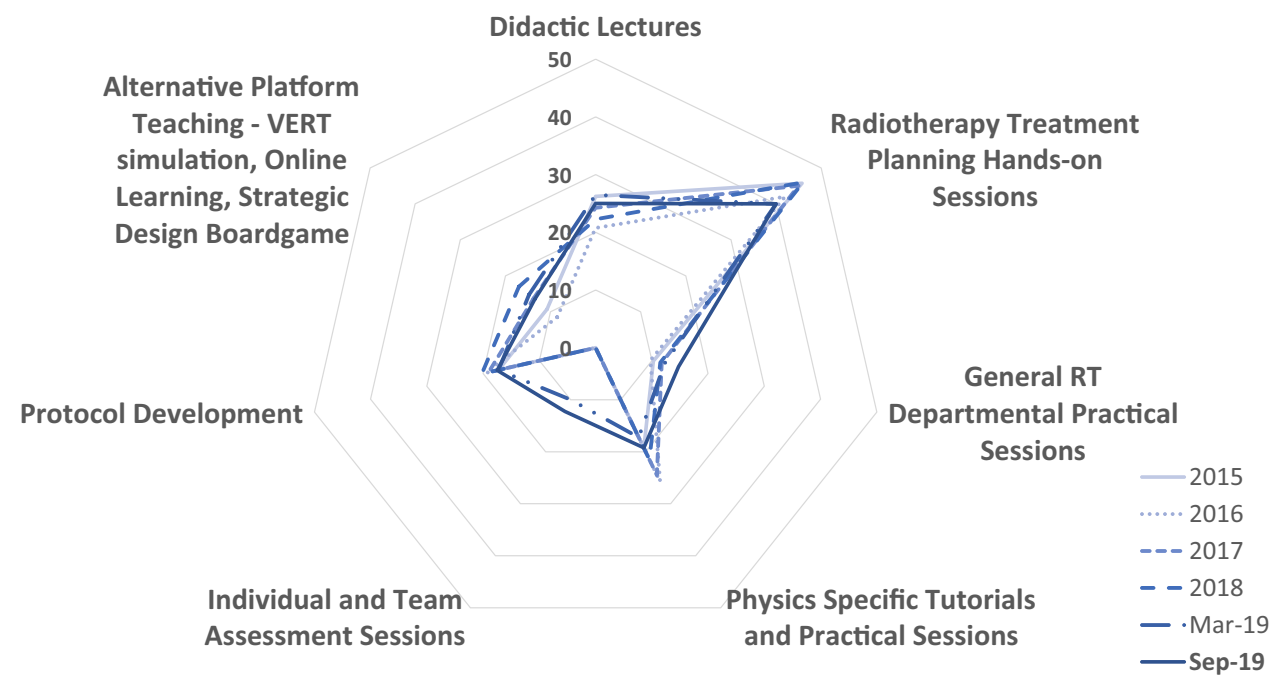

\section{Geographic Reach of the Programme}

Thirteen teams from Africa attended the courses offered between 2015 and 2019, with two hospitals sending two teams each. Each team consisted of one radiation oncologist, one medical physicist and one RTT (Table 1).

In addition, any unfilled course places were filled with teams from seven hospitals predominantly in the state sector in South Africa, in support of local professional skills development initiatives. Where extra individual spaces were available for a given course, these were accommodated in mixed multi-disciplinary teams and some of the attendees included international staff on IAEA fellowships or international registrar appointments. The countries of origin of these individuals included Republic of the Congo, Democratic Republic of the Congo, Caribbean Islands and Kenya.

Analysis of the DIRAC database [9] shows that all but one team had access to MV radiotherapy machines no more than 10 years old (Table 1). However, long delays between attending the course and the actual installation was observed for several of the teams, limiting the immediate impact of the training course.

At the time of the programme assessment (2021), only one team had not yet managed to proceed with installation of new equipment.

\section{Programme Evaluation and Participant Feedback}

Paper-based course evaluations were performed in 2015 and 2016. These were replaced with Google form surveys from 2016 onwards. Survey results included comments on the academic programme, technical aspects of the course, and most valuable learning points.

In terms of programme content and design, consistent feedback was received between 2015 and 2018 that the 3-week period was insufficient to cover all clinical sites. Participants also felt they required more time to practice the contouring and planning skills. Based on this, a decision
Table 1 International teams 2015-2019

\begin{tabular}{lllll}
\hline City & Country & Course date & $\begin{array}{l}\text { DIRAC MV/MeV equip- } \\
\text { ment status 2021 }\end{array}$ & $\begin{array}{l}\text { Some equip- } \\
\text { ment } \leq 10 \text { years* }\end{array}$ \\
\hline Harare & Zimbabwe & 2015 & 4 & $\mathrm{Y}$ \\
Bulawayo & Zimbabwe & 2015 & 1 & $\mathrm{Y}$ \\
Kumasi & Ghana & 2015 & 1 & $\mathrm{Y}$ \\
Accra & Ghana & 2015 & 1 & $\mathrm{Y}$ \\
Yaounde & Cameroon & 2016 & 0 & $\mathrm{~N}$ \\
Addis Ababa & Ethiopia & 2016,2018 & 1 & $\mathrm{Y}$ \\
Misrata & Libya & 2017 & 1 & $\mathrm{Y}$ \\
Dar Es Salaam & Tanzania & 2018,2018 & 2 & $\mathrm{Y}$ \\
Abuja & Nigeria & 2019 & 2 & $\mathrm{Y}$ \\
Lagos & Nigeria & 2019 & 2 & $\mathrm{Y}$ \\
Lusaka & Zambia & 2019 & 1 & $\mathrm{Y}$ \\
\hline
\end{tabular}

*DIRAC 2020[9] 
was taken in 2018 to reduce the clinical sites to three (prostate, cervix and breast) to allow for more hands-on practice time. New courses were planned to be developed in 2020 to address the skills required for the treatment of head and neck and neurological cancers.

The feedback also highlighted four dominant themes relating to learning points:

- Challenging hierarchical structures in home centres

- Appreciation of the teamwork principle

- Feelings of empowerment

- Financial constraint concerns

The first dominant theme was that of challenging the hierarchical structures or 'traditional structure' and was expressed as follows:

- Higher powers that might not agree to the changes I might want to take place. But I strongly believe there will be a way, though it may take time.

- Understanding of policy makers but one can always go around this with the acquire (d) knowledge.

- My capacity/opportunity to influence change in the running of my unit.

- The hospital management may delay decisions.

The second dominant theme included both the challenge of implementing teamwork and the appreciation of the need for teamwork, including comments such as "as long as there is teamwork" and "resistance from colleagues". The desire towards "engaging all specialists towards teamwork" and the need for "support from other staff". This was also seen as a big positive learning with comments such as: "It was very valuable to see how the three professions complement each other. There was a clear distinction amongst them in a very balanced manner i.e. There wasn't inferiority, every lecture was balanced and well suited for all three professions" and "To work in team, decide in team, agree the responsibilities in team and put the patient in the centre of our clinical interest."

The third theme that emerged was the feeling of empowerment expressed by participants in the skills learnt and the philosophy of inter-disciplinary practice experienced during the course. Participants also specifically reflected about knowledge imparted and their reactions within in the learning process and what this experience meant to them. Several participants voiced their feelings of empowerment - witness the direct quotes below:

- Knowing yourself in terms of knowledge

- Gift you are given is the gift only you can accept

- We are the change makers

- Power of knowledge, enlightening

\section{- Learning from peers was excellent}

The fourth theme was related to financial constraints. Again this was tempered by the sense of optimism and finding a way forward as illustrated by the comment "we have not yet finished installations of our linear accelerators, but we will use the time we have to develop our protocols (Physics, RTTs \& RO)".

\section{Discussion}

The $\mathrm{A} 2 \mathrm{C}$ training programme is a hybrid e-learning and on-site radiotherapy training programme giving qualified radiotherapy professionals access to 17 days of practical skills training focusing on 3D conformal radiotherapy. The programme makes use of a wide variety of teaching methods and equipment, including pre-course e-learning, free-thinking design exercises, didactic learning, hands-on computer sessions, virtual simulation training and departmental demonstration sessions.

An assessment of the time utilization during the programme shows a clear emphasis on hands-on practical training on the TPSs, with up to $52 \mathrm{~h}$ (2019) of each course dedicated to actively contouring or planning clinical cases. All cases are selected to represent staging and clinical presentation often seen in the region. This time allocation also includes the individual and team assessment challenges introduced in 2019. An average of $18 \mathrm{~h}$ per course is spent on clinical protocol development, with a further 3 months of remote support to teams to continue developing protocols for their own departments. The assessment also showed that pre-course online learning on the LäraNära platform is only successful if active progress tracking mechanisms are put in place, with an improvement from 33.8 to $76 \%$ in test completion rate reported after a formal progress tracking system was implemented in 2019.

Thirteen teams from eight countries in Africa attended the course between 2015 and 2019, with each team including a radiation oncologist, medical physicist and radiation therapy technologist. The DIRAC database shows that 12 out of the 13 teams now have access to some equipment less than 10 years old (2021), indicating successful targeting of teams in the process of transitioning to 3DCRT. However, the delay between attending the course and installation of equipment is a major concern in terms of knowledge retention and application of new skills learned, resulting in some centres sending a second team for training, or requesting assistance with ongoing training.

In terms of participant feedback, the main concern raised by participants is the need to challenge traditional hierarchical structures within their departments to facilitate better collaboration between all three professions. Even though 
participants acknowledged the potential gain in encouraging teamwork within their departments, they were concerned about their ability to implement the strategies.

In the light of the current travel challenges being experienced globally because of the Covid-19 pandemic, the programme is being redesigned to allow for remote teaching of most components of the course. Thus, the first remote courses were designed and run to address the needs and challenges experienced in 2020, with the full remote implementation of the 3DCRT course planned for the end of 2021.

Funding The $\mathrm{A} 2 \mathrm{C}$ radiotherapy training programme is a collaboration between the University of Cape Town, Cape Peninsula University of Technology and Varian Medical Systems.

\section{Data Availability N/A}

Code Availability N/A

\section{Declarations}

Ethics Approval The study is approved by the Human Research Ethics Committee of the University of Cape Town (HREC REF: 829/2019).

Consent for Publication Informed consent was obtained from all participants to use feedback and comments for research and publication.

Conflict of Interest H Burger acts as consultant to Varian Medical Systems and is the University of Cape Town coordinator for the A2C training programme. J Groll, JM Valentim and S Berz are employed by Varian Medical Systems.

\section{References}

1. (IARC), World Health Organization International Agency for Research on Cancer (2018) GLOBOCAN 2018: Estimated cancer incidence, mortality and prevalence worldwide in 2018. https:// gco.iarc.fr/today/home. Accessed 28 April 2018

2. (IARC), World Health Organization International Agency for Research on Cancer (2021) GLOBOCAN 2020: Estimated cancer incidence, mortality and prevalence worldwide in 2020. https:// gco.iarc.fr/today/fact-sheets-populations. Accessed 10 Feb 2021

3. Sung H, Ferlay J, Siegel RL, Laversanne M, Soerjomataram I, Jemal A, Bray F (2021) Global cancer statistics 2020: GLOBOCAN estimates of incidence and mortality worldwide for 36 cancers in 185 countries. CA Cancer J Clin. https://doi.org/10.3322/ caac. 21660
4. Pistenmaa DA, Dosanjh M, Amaldi U, Jaffray D, Zubizarreta E, Holt K, Lievens Y, Pipman Y, Norman C, Coleman. (2018) Changing the global radiation therapy paradigm. Radiother Oncol 128(3):393-399

5. Omonisi AE, Liu B, Parkin DM (2020) Population-based cancer registration in sub-Saharan Africa: its role in research and cancer control. JCO Global Oncology 6:1721-1728

6. Atun R, Jaffray DA, Barton MB, Bray F, Baumann M, Vikram B, Hanna TP, Knaul FM, Lievens Y, Lui TYM (2015) Expanding global access to radiotherapy. Lancet Oncol 16(10):1153-1186

7. Bishr MK, Zaghloul MS (2018) Radiation therapy availability in Africa and Latin America: two models of low and middle income countries. Int J Rad Onc* Biol* Physics 102 (3):490-498

8. Levitt SH, Leer J-W (1996) The role of radiotherapy in Sweden: a landmark study by the Swedish council on technology assessment in health care. Acta Oncol 35(8):965-966

9. (IAEA), International Atomic Energy Agency (2021) Directory of radiotherapy centres. https://dirac.iaea.org/Query/Map2? mapId=0. Accessed 29 March 2021

10. Abdel-Wahab M et al (2013) Status of radiotherapy resources in Africa: an International Atomic Energy Agency analysis. Lancet Oncol 14(4):e168-e175

11. (IAEA), International Atomic Energy Agency (2017) Directory of radiotherapy centres (DIRAC). https://dirac.iaea.org/Query/ Map2? mapId=0. Accessed 24/04/2017

12. Zubizarreta EH, Fidarova E, Healy B, Rosenblatt E (2015) Need for radiotherapy in low and middle income countries-the silent crisis continues. Clin Oncol 27(2):107-114

13. Rodin D, Longo J, Sherertz T, Shah MM, Balagun O, Wendling N, Van Dyk J, Coleman CN, Xu MJ, Grover S (2017) Mobilising expertise and resources to close the radiotherapy gap in cancer care. Clin Oncol 29 (2):135-140

14. Degerfält J, Sjöstedt S, Fransson P, Kjellén E, Werner MU (2017) E-learning programs in oncology: a nationwide experience from 2005 to 2014. BMC Res Notes 10(1):1-14

15. Parkes J, Burger H, Wyrley-Birch B, Valentim J-M, Groll J (2016) O56. The Cape Town Access to Care course, our ongoing teaching journey. Eur J Med Phys. Abstract only. 32(S2):159-160. https://doi.org/10.1016/j.ejmp.2016.07.064

16. Trauernicht C, Parkes J, Burger H, Wyrley-Birch B, Valentim JM, Groll J (2016) The Cape Town Access to Care Cours: bridging the African radiotherapy training gap. International Conference on Medical Physics. Med Phys Int J. Abstract only. 4(2):358

17. Van Dyk J (ed) (2013) The modern technology of radiation oncology, volume 3: a compendium for medical physicists and radiation oncologists. Medical Physics Publishing, Madison, pp 361-412

18. SSM (2011) Report from SSM's scientific council on ionizing radiation within oncology, 2010. Swedish Radiation Safety Authority, Sweden

Publisher's Note Springer Nature remains neutral with regard to jurisdictional claims in published maps and institutional affiliations. 